\title{
Properly homotopic nontrivial planes are isotopic
}

\author{
by
}

Bobby Neal Winters (Pittsburg, Kan.)

\begin{abstract}
It is proved that two planes that are properly homotopic in a noncompact, orientable, irreducible 3-manifold that is not homeomorphic to $\mathbb{R}^{3}$ are isotopic. The endreduction techniques of E. M. Brown and C. D. Feustal and M. G. Brin and T. L. Thickstun are used.
\end{abstract}

Introduction. In this paper it is proved that two planes that are properly homotopic in a noncompact, orientable, irreducible 3-manifold that is not homeomorphic to $\mathbb{R}^{3}$ are isotopic. The end-reduction techniques of Brown-Feustal and Brin-Thickstun are used.

It is not uncommon among those who study noncompact 3-manifolds to consider the end-irreducible and eventually end-irreducible cases as a starting point. These cases, while quite far from being general, do occur often enough to be useful. In recent years a technique known as "endreduction" has been used to extend from the eventually end-irreducible case to the general case.

The technique of end-reduction was used by Brown and Feustel in [BF] to prove that if there is a "nontrivial" mapping of $\mathbb{R}^{2}$ in a noncompact 3-manifold $W$, then $W$ must contain a "nontrivial" embedded plane as well. This result had been proved in [BBF] for eventually end-irreducible $W$.

In [BT] Brin and Thickstun recognized that given a noncompact 3-manifold $W$ and a compact $K \subset W$ an eventually end-irreducible 3manifold $W_{K}$ could be associated with $(W, K)$. By using the properties of the eventually end-irreducible 3 -manifold $W_{K}$ for increasingly large $K$ and piecing things together nicely, they were able to obtain results in a more general case.

Following the approach of [BT], this author proved in [W] that if $W$ is a noncompact, orientable, irreducible 3-manifold that is not homeomorphic to $\mathbb{R}^{3}$, and $P$ and $Q$ are planes that are nontrivial in $W$ with $P$ properly

1991 Mathematics Subject Classification: 57M99, 57N10. 
homotopic to $Q$ and $P \cap Q=\emptyset$, then $P$ and $Q$ are parallel in $W$. This paper, which is a sequel to $[\mathrm{W}]$, follows the methods of $[\mathrm{BF}]$ more closely, however.

Definitions. A plane (annulus, circle, arc, 2-sphere, disk) is a space homeomorphic to $\mathbb{R}^{2}\left(\mathbb{S}^{1} \times I, \mathbb{S}^{1}, I, \mathbb{S}^{2}, \mathbb{D}^{2}\right)$.

Suppose that $X, Y$, and $Z$ are topological spaces.

If $f: X \rightarrow Y$ is a map and $f^{-1}(K)$ is compact for every compact $K \subset Y$, then $f$ is said to be proper. If $X \subset Y$ and $X \cap K$ is compact for every compact $K \subset Y$, then $X$ is proper in $Y$. (This is equivalent to the inclusion map $X \rightarrow Y$ being proper.) If $X \subset Y$ and $X$ and $Y$ are $n$ - and $(n+1)$ - manifolds, respectively, then we say that $X$ is properly embedded in $Y$ when $X$ is proper in $Y$ and $X \cap \partial Y=\partial X$.

Suppose that $X$ and $Y$ are subspaces of $Z$. We say that $X$ traps $Y$ if there is no proper homotopy $h: Y \times I \rightarrow Z$ such that $h(y, 0)=y$ for every $y \in Y$ and $h(Y \times 1) \cap X=\emptyset$.

Let $h: X \times I \rightarrow X$ be a proper map. For $t \in I$, define $h_{t}: X \rightarrow X$ by $h_{t}(x)=h(x, t)$. If $h_{0}=1_{W}$ and $h_{t}$ is a homeomorphism for every $t \in I$, then we say that $h$ is an isotopy of $X$.

We let $\sharp(X)$ denote the number of path components of the space $X$.

Suppose that $W$ is a noncompact 3-manifold.

If $P \subset W$ is a plane, then we say that $P$ is nontrivial in $W$ when $P$ is proper in $W$ and there is a compact subset of $W$ that traps $P$.

An exhaustion or exhausting sequence for $W$ is a function $V$ from $\mathbb{N}$ to the set of compact 3-submanifolds of $W$ such that $W=\bigcup_{n \in \mathbb{N}} V(n)$ and $V(n) \subset V(n+1)-\operatorname{Fr}(V(n+1))$. Traditionally we put $V_{n}=V(n)$.

If there is an exhausting sequence $V$ for $W$ such that $\operatorname{Fr}\left(V_{n}\right)$ is incompressible in $W$ for every $n \in \mathbb{N}$, then we say that $W$ is end-irreducible.

If there is a compact subset $K \subset W$ and an exhaustion $V$ for $W$ such that $K \subset V_{1}-\operatorname{Fr}\left(V_{1}\right)$ and $\operatorname{Fr}\left(V_{n}\right)$ is incompressible in $W-K$ for every $n \in \mathbb{N}$, then $W$ is said to be end-irreducible rel $K$. If $W$ is end-irreducible rel $K$ for some compact $k \subset W$, then $W$ is said to be eventually end-irreducible.

\section{Some results about compact 3-manifolds}

Lemma 1. Let $M$ be a compact 3-manifold and let $T \subset \partial M$ be a compact 2-manifold with at least two components. For $i=1,2$, let $A_{i}$ be an annulus that is properly embedded and incompressible in $M$ with each component of $\partial A_{i}$ contained in a different component of $T$. Suppose that if $J$ is a component of $A_{1} \cap A_{2}$, then $J$ is a circle in $M-\partial M$ that is noncontractible in $M$. If $D$ is a compressing disk for $T$ in $M$, then there is a compressing disk $D^{\prime}$ for $T$ in $M$ such that $D^{\prime} \cap\left(A_{1} \cup A_{2}\right)=\emptyset$. 
Pr o of. Suppose that $D$ is a compressing disk for $T$ in $M$. By Lemma 2 of $[\mathrm{W}]$ (in which the assumption of irreducibility in the hypothesis may be dropped), we may assume that $D \cap A_{1}=\emptyset$. Now choose such a disk $D$ with $\sharp\left(D \cap A_{2}\right)$ minimal. By standard arguments involving innermost disks and incompressibility, we may assume that no component of $D \cap A_{2}$ is a circle.

To get a contradiction, suppose that $\alpha$ is an arc component of $D \cap A_{2}$. Let $D_{\alpha}$ be a disk that is separated off $D$ by $\alpha$. We may choose $\alpha$ so that $D_{\alpha} \cap A_{2}=\alpha$. Since each component of $\partial A_{2}$ is in a different component of $T$, it follows that $\alpha$ is a separating arc of $A_{2}$. Let $E_{\alpha}$ be the disk separated off $A_{2}$ by $\alpha$. Then $D_{\alpha} \cup E_{\alpha}$ is a disk. Let $J=\partial\left(D_{\alpha} \cup E_{\alpha}\right)$.

If $J$ is noncontractible in $T$, then there is a compressing disk $D^{\prime}$ for $T$ in $M$ that is parallel to $D_{\alpha} \cup E_{\alpha}$ with $D^{\prime} \cap\left(A_{1} \cup A_{2}\right)=\emptyset$. On the other hand, suppose that there is a disk $E \subset T$ with $\partial E=J$. Then we may slide $\partial D$ along $E$ to move it past $A_{2}$ and remove an arc of $D \cap A_{2}$ while introducing a circle of $D \cap A_{2}$. This circle can be removed by standard methods. We have reduced $\sharp\left(D \cap A_{2}\right)$, which contradicts minimality. This ends the proof.

LEMma 2. Let $M \subset N$ be compact, irreducible 3-manifolds such that each component of $\operatorname{cl}(N-M)$ meets $\partial N$. Suppose that $A_{0}$ and $A_{1}$ are annuli that are incompressible and properly embedded in $M$ with $\partial A_{0}=\partial A_{1}$. If $A_{0}$ and $A_{1}$ are homotopic in $N$, then $A_{0}$ and $A_{1}$ are isotopic in $M$ by an isotopy fixed on $\partial M$.

Proof. Move $A_{1}$ by an isotopy of $M$ fixed on $\partial M$ so that $\sharp\left(A_{0} \cap A_{1}\right)$ is minimal and $A_{0}$ meets $A_{1}$ transversely.

Suppose that $J$ is a component of $\left(A_{0} \cap A_{1}\right)-\partial A_{0}$ that is contractible in either $A_{0}$ or $A_{1}$. It is easy to argue using the incompressibility of $A_{0}$ and $A_{1}$ that $J$ must be contractible in both $A_{0}$ and $A_{1}$. Let $D \subset A_{1}$ be a disk with $J=\partial D$. Without loss of generality, we may assume that $(D-\partial D) \cap A_{0}=\emptyset$. Let $E \subset A_{0}$ be the disk with $\partial E=\partial D$. Since $M$ is irreducible and $D \cup E$ is a 2-sphere, there is a 3 -ball $B \subset M$ with $\partial B=D \cup E$. We may use $B$ to reduce \# $\left(A_{0} \cap A_{1}\right)$ by an isotopy of $M$. Therefore, we may assume that no component of $\left(A_{0} \cap A_{1}\right)$ is contractible in either $A_{0}$ or $A_{1}$. Consequently, if $F$ is the closure of a component of $A_{0}-A_{1}$ or $A_{1}-A_{0}$, then $F$ is not a disk.

Suppose that $F$ is the closure of a component of $A_{0}-A_{1}$ or $A_{1}-A_{0}$.

By Proposition 5.4 of [Wa], there is a product $F \times I \subset N$ such that $F \times 0 \subset A_{0},(\partial F \times I) \cup(F \times 1) \subset A_{1}$, and $(F \times(0,1)) \cap\left(A_{0} \cup A_{1}\right)=\emptyset$.

Since $\partial(F \times I) \subset M$, either $F \times I \subset M$ or the interior of $F \times I$ contains a component of $\operatorname{cl}(N-M)$. Since each component of $\operatorname{cl}(N-M)$ meets $\partial N$, it follows that $F \times I \subset M$.

Since $\sharp\left(A_{0} \cap A_{1}\right)$ is minimal, we have $F \times 0=A_{0}$ and $(\partial F \times I) \cup(F \times 1)$ $=A_{1}$. Therefore $A_{1}$ is isotopic in $M$ to $A_{0}$ by an isotopy fixed on $\partial M$. 
Handle moves respecting planes. Beginning now and for the rest of the paper, let $W$ be a connected, noncompact, orientable, irreducible 3 -manifold that is not homeomorphic to $\mathbb{R}^{3}$, and let $P$ and $Q$ be planes that are nontrivial in $W$.

In what follows, we will at times need to do handle moves along compressing 1-, 2-, and 3-handles. In particular, suppose that $M \subset W$.

First suppose that there is a properly embedded disk $D \subset M$ with $\partial D$ a noncontractible circle in $\operatorname{Fr}(M)$. Let $H$ be a regular neighborhood of $D$ in $M$. Then $H$ is a compressing 1-handle for $M$. Let $M(H)=\operatorname{cl}(M-H)$. We say that $M(H)$ is obtained from $M$ by removing the 1-handle $H$.

Suppose that $D \subset \operatorname{cl}(W-M)$ is a properly embedded disk with $\partial D$ noncontractible in $\operatorname{Fr}(M)$. Let $H$ be a regular neighborhood of $D$ in $\operatorname{cl}(W-M)$. Then we say that $H$ is a compressing 2-handle for $M$. Let $M(H)=M \cup H$. We say that $M(H)$ is obtained from $M$ by adding the 2-handle $H$.

Suppose that $S$ is a 2-sphere in $\operatorname{Fr}(M)$ that bounds a 3 -ball $H$ in $\operatorname{cl}(W-M)$. Then $H$ is a compressing 3 -handle for $M$. Let $M(H)=M \cup H$. Then $M(H)$ is said to be obtained from $M$ by adding the 3 -handle $H$.

When $H$ is a compressing 1-, 2-, or 3-handle for $M$, then we say that $H$ is a compressing handle for $M$.

Suppose that $H_{1}$ is a compressing handle for $M$. Suppose that $H_{2}$ is a compressing handle for $M\left(H_{1}\right)$. Define $M\left(H_{1}, H_{2}\right)=M\left(H_{1}\right)\left(H_{2}\right)$. Assume that $M\left(H_{1}, \ldots, H_{k-1}\right)$ has been defined and let $H_{k}$ be a compressing handle for $M\left(H_{1}, \ldots, H_{k-1}\right)$. Define $M\left(H_{1}, \ldots, H_{k}\right)=M\left(H_{1}, \ldots, H_{k-1}\right)\left(H_{k}\right)$. Then we say that $H_{1}, \ldots, H_{k}, \ldots$ is a sequence of compressing handles in $W$ for $M$.

Let $K \subset M$ and $F \subset W$. Suppose that $H_{1}, \ldots, H_{\nu}$ is a sequence of disjoint compressing 1-handles in $W-K$ for $M$ such that

(1) $H_{i} \cap F=\emptyset$ and $H_{i} \cap K=\emptyset$ for $1 \leq i \leq \nu$, and

(2) $\operatorname{Fr}\left(M\left(H_{1}, \ldots, H_{\nu}\right)\right)$ is incompressible in $W-K$.

Then we say that $M$ can be compressed in $W-K$ to $M\left(H_{1}, \ldots, H_{\nu}\right)$ by removing 1-handles that miss $F$.

Lemma 3. Suppose that $K \subset W$ is a compact 3-manifold that traps $P$ and meets $P$ in a single disk. Suppose that $L \subset W$ is compact. Then there is a compact, connected 3-manifold $M \subset W$ with $K \cup L \subset M-\operatorname{Fr}(M)$ such that $P \cap M$ is a single disk and $M$ can be compressed in $W-K$ by removing 1-handles that miss $P$.

Proof. The proof is essentially the first two paragraphs of the proof of Lemma 3 of $[\mathrm{W}]$, which owes much to Lemma 1.1 of $[\mathrm{BF}]$. We repeat it here for the convenience of the reader. 
Let $M \subset W$ be a compact, connected 3-manifold with $K \cup L \subset M-$ $\operatorname{Fr}(M)$ such that $P \cap M$ is a single disk. There is a sequence $H_{1}, \ldots, H_{\nu}$ of compressing 1-, 2-, and 3-handles in $W-K$ for $M$ such that if $M^{*}=$ $M\left(H_{1}, \ldots, H_{\nu}\right)$, then $\operatorname{Fr}(M)^{*}$ is incompressible in $W-K$. We may argue using Lemma 1 and the fact that $W-K$ is irreducible that $H_{1}, \ldots, H_{\nu}$ may be chosen so as to not intersect $P$. We choose $M$ so that, with respect to the indicated properties, $H_{1}, \ldots, H_{\nu}$ contains the fewest possible 2-handles.

We claim that $H_{1}, \ldots, H_{\nu}$ has no 2 -handles (and therefore no 3 -handles). Let $k$ be the least integer such that $H_{k}$ is a 2-handle. We may choose $H_{k}$ so that $H_{k} \cap H_{i}$ is a subproduct of the 1-handle structure of $H_{i}$ for $1 \leq i \leq k-1$. Let $H=\bigcup_{i=1}^{k-1} H_{i}$ and let $H_{1}^{\prime}, \ldots, H_{\mu}^{\prime}$ be the components of $\operatorname{cl}\left(H-H_{k}\right)$. Let $M^{\prime}=M \cup H_{k}$. Then $H_{i}^{\prime}$ is a 1 -handle for $M^{\prime}$ for $1 \leq i \leq \mu$. Note that $M^{\prime}\left(H_{1}^{\prime}, \ldots, H_{\mu}^{\prime}, H_{k+1}, \ldots, H_{\nu}\right)=M^{*}$. Since $H_{1}^{\prime}, \ldots, H_{\mu}^{\prime}, H_{k+1}, \ldots, H_{\nu}$ has fewer 2-handles, this contradicts the minimality assumption. This ends the proof.

Let $V$ be an exhaustion for $W$. For $n \in \mathbb{N}$, let $G_{n}=\operatorname{cl}\left(V_{n+1}-V_{n}\right)$, $A_{n}=P \cap G_{n}$, and $B_{n}=Q \cap G_{n}$. Suppose that $A_{n}$ and $B_{n}$ are incompressible annuli that are properly embedded in $G_{n}$ and meet both $\operatorname{Fr}\left(V_{n+1}\right)$ and $\operatorname{Fr}\left(V_{n}\right)$, and suppose that each component of $A_{n} \cap B_{n}$ is a circle in $G_{n}-\partial G_{n}$ that is noncontractible in $G_{n}$. Note that $P \cap V_{1}$ and $Q \cap V_{1}$ are necessarily disks.

Lemma 4. Suppose that $L \subset W$ is compact. There is a compact, connected 3-manifold $M \subset W$ such that $V_{1} \cup L \subset M-\operatorname{Fr}(M), P \cap M$ and $Q \cap M$ are both disks, and $M$ can be compressed in $W-V_{1}$ by removing 1-handles that miss $P \cup Q$.

Re mark. The proof that follows is a modification of the first two paragraphs of the proof of Lemma 3 of [W], which itself owes much to Lemma 1.1 of $[\mathrm{BF}]$.

Proof of Lemma 4. Note that, for $n \in \mathbb{N}, P \cap V_{n}$ and $Q \cap V_{n}$ are both single disks.

Let $M$ be a compact, connected 3-manifold with $V_{1} \cup L \subset M-\operatorname{Fr}(M)$ such that $P \cap M$ and $P \cap Q$ are single disks, and $P \cap \operatorname{cl}\left(M-V_{1}\right)$ and $Q \cap \operatorname{cl}\left(M-V_{1}\right)$ are annuli that are incompressible in $\operatorname{cl}\left(M-V_{1}\right)$, meet both $\operatorname{Fr}(M)$ and $\operatorname{Fr}\left(V_{1}\right)$, and intersect one another in circles that are noncontractible in $\operatorname{cl}\left(M-V_{1}\right)$. So far $M=V_{m}$ for some $m \geq 2$ would satisfy these conditions.

Let $H_{1}, \ldots, H_{\nu}$ be a compressing sequence of 1-, 2-, and 3-handles for $M$ which miss $P \cup Q$. By Lemma 1, such a compressing sequence exists. Choose $M$ so that $H_{1}, \ldots, H_{\nu}$ has the fewest possible 2-handles. The rest of the proof proceeds as in the latter part of the proof of Lemma 3. 
Proper homotopies between planes. Beginning now and for the rest of the paper, let $f: \mathbb{R}^{2} \times I \rightarrow W$ be a proper map such that $f \mid \mathbb{R}^{2} \times i$ is an embedding for $i \in \partial I$, and $f\left(\mathbb{R}^{2} \times 0\right)=P$ and $f\left(\mathbb{R}^{2} \times 1\right)=Q$.

For this section, suppose that $K \subset W$ is a compact, connected 3-manifold that traps both $P$ and $Q$ and meets $P$ in a single disk. Also assume that no component of $\operatorname{Fr}(K)$ is a 2-sphere. Since $K$ traps $P$, it can be argued that there is no 3-ball $B \subset W$ with $K \subset B-\partial B$. It now follows that $W-K$ is irreducible and that $\pi_{2}(W-K)=0$. We may also argue that $P-K$ is incompressible in $W-K$.

Let $\Delta \subset \mathbb{R}^{2}$ be a disk and let $\Lambda=\operatorname{cl}\left(\mathbb{R}^{2}-\Delta\right)$; then $\Lambda$ is homeomorphic to $\mathbb{S}^{1} \times[0, \infty)$, a half open annulus. Since $f$ is proper, we may choose $\Delta$ so that $f(\Lambda \times I) \subset W-K$. It follows that $P \cap K \subset f(\Delta \times 0)-f(\partial \Delta \times 0)$. So $f(\partial \Lambda \times 0)=f(\partial \Delta \times 0)$ is noncontractible in $W-K$.

Let $N$ be a compact, connected 3-manifold in $W$ such that $K \subset N-$ $\operatorname{Fr}(N)$ and $\operatorname{Fr}(N)$ is in general position with respect to $P$ and $Q$. Suppose that $f(\Delta \times I) \subset N-\operatorname{Fr}(N)$ and that $P \cap N$ is a single disk. Let $M^{\prime}$ be a compact 3-manifold obtained from $N$ by removing 1-handles that miss $P$ and are transverse to $Q$. Suppose that there is a component $M$ of $M^{\prime}$ such that $K \subset M-\operatorname{Fr}(M)$ and $\operatorname{Fr}(M)$ is incompressible in $W-K$. Let $D=\operatorname{cl}(\operatorname{Fr}(M)-\operatorname{Fr}(N))$. Then each component of $D$ is a disk.

Since $\operatorname{Fr}(M)$ is incompressible in $W-K$, it follows that

$$
\operatorname{ker}\left(\pi_{1}(\operatorname{Fr}(M)) \rightarrow \pi_{1}(W-K)\right)
$$

is trivial. It is also easy to argue that

$$
\pi_{2}(\operatorname{Fr}(M))=\pi_{2}((W-K)-\operatorname{Fr}(M))=0 .
$$

In Lemma 5, we make use of the techniques used by Hempel in the proof of Lemma 6.5 of [He]. For the convenience of the reader, we reproduce the part of Hempel's language that we need here without proof.

Let $g: \Lambda \times I \rightarrow W-K$ be a proper map. We will wish at times in the proof of Lemma 5 to obtain a proper map $g_{1}: \Lambda \times I \rightarrow W-K$ that agrees with $g$ except on the interior of some closed 3-ball contained in $\Lambda \times I$. These modifications are in the from of three "moves" listed below. We refer the reader to the body of the proof of Lemma 6.5 of [He] for the proof and more specific details of the respective modifications. However, note that $(*)$ and $(* *)$ above satisfy all of the algebraic hypotheses that the proof requires.

Without loss of generality, we may assume that $g^{-1}(\operatorname{Fr}(M))$ is a 2-sided, compact 2-manifold. Let $F$ be a component of $g^{-1}(\operatorname{Fr}(M))$.

Move 1 . Suppose that $F$ is a 2 -sphere that bounds a 3-ball $B^{\prime} \subset \Lambda \times I$. Then we change the definition of $g$ on a regular neighborhood $B \subset(\Lambda \times I)$ $-\partial(\Lambda \times I)$ of $B^{\prime}$ to obtain a new map $g_{1}$ that agrees with $g$ off $B-\partial B$ and $g_{1}^{-1}(\operatorname{Fr}(M))=g^{-1}(\operatorname{Fr}(M))-B$. 
Move 2. Suppose that $F$ is a disk that is parallel in $\Lambda \times I$ to a disk in $\partial(\Lambda \times I)$ by a parallelism $B^{\prime}$. We may change the definition of $g$ on a regular neighborhood $B$ of $B^{\prime}$ to obtain a map $g_{1}$ that agrees with $g$ off $B-\partial B$ such that $g_{1}^{-1}(\operatorname{Fr}(M))=g^{-1}(\operatorname{Fr}(M))-B$.

Move 3. Suppose that there is a disk $D$ in $\Lambda \times I$ with $\partial D=D \cap$ $g^{-1}(\operatorname{Fr}(M)) \subset F$ such that $\partial D$ is contractible in $F$. Then there is a ball $B \subset(\Lambda \times I)-\partial(\Lambda \times I)$ which contains $D$ and intersects $g^{-1}(\operatorname{Fr}(M))$ precisely in a regular neighborhood of $\partial D$ in $F$. We may change the definition of $g$ on $B$ to obtain a map $g_{1}$ that agrees with $g$ off $B-\partial B$ such that $g_{1}^{-1}(\operatorname{Fr}(M))$ may be obtained from $g^{-1}(\operatorname{Fr}(M))$ by removing the interior of an annulus regular neighborhood of $\partial D$ in $F$ and capping off the two resulting circles with a pair of disjoint disks in $\Lambda \times I$ which intersect $g^{-1} \operatorname{Fr}(M)$ precisely in the boundaries of the disks.

We will also borrow from Hempel the measure of complexity of maps that he uses. For $i=2,1,0,-1, \ldots$, let $c_{i}(g)$ be the number of components of $g^{-1}(\operatorname{Fr}(M))$ that have Euler characteristic equal to $i$. Let $c(g)=$ $\left(\ldots, c_{-1}, c_{0}(g), c_{1}(g), c_{2}(g)\right)$.

Lemma 5. (1) There is a proper map $g: \Lambda \times I \rightarrow W-K$ such that $g|\partial(\Lambda \times I)=f| \partial(\Lambda \times I)$ and if $F$ is a component of $g^{-1}(\operatorname{Fr}(M))$, then $F$ is 2 -sided, is not a 2 -sphere, and the inclusion induced map $\pi_{1}(F) \rightarrow \pi_{1}(\Lambda \times I)$ is injective.

(2) There is a proper map $h: \Lambda \times I \rightarrow W-K$ such that $h \mid \Lambda \times \partial I=$ $f \mid \Lambda \times \partial I$ and if $F$ is a component of $h^{-1}(\operatorname{Fr}(M))$, then $F$ is 2-sided, is not a 2-sphere, the inclusion induced map $\pi_{1}(F) \rightarrow \pi_{1}(\Lambda \times I)$ is injective, and no component of $F \cap(\partial \Lambda \times I)$ is a circle.

Proof. We mimic the proof of Lemma 6.5 of [He] with obvious modifications.

To prove part (1), let $g: \Lambda \times I \rightarrow W-K$ be a proper map such that $g|\partial(\Lambda \times I)=f| \partial(\Lambda \times I)$ and let $F=g^{-1}(\operatorname{Fr}(M))$. We may choose $g$ so that $F$ is 2 -sided and $g$ differs from $f$ only by repeated modifications by Move 1 and Move 3. It may be that $g=f$. Choose $g$ so that $c(g)$ is minimal when taken in lexicographic order. It is easy to check that no component $F^{\prime}$ of $F$ is a 2-sphere and that if $F^{\prime}$ is a component of $F$, then $\pi_{1}\left(F^{\prime}\right) \rightarrow \pi_{1}(\Lambda \times I)$ is injective.

To prove part (2), let $h: \Lambda \times I \rightarrow W-K$ be a proper map such that $h|\Lambda \times \partial I=f| \Lambda \times \partial I$. Let $F=h^{-1}(\operatorname{Fr}(M))$. We may assume by part (1) that if $F^{\prime}$ is a component of $F$, then $F^{\prime}$ is not a 2-sphere and $\pi_{1}\left(F^{\prime}\right) \rightarrow \pi_{1}(\Lambda \times I)$ is injective. We allow $h$ to differ from $f$ by successive modifications by Move 2. Choose $h$ among such maps so that $\#(F)$ is minimal.

We claim that no component of $F \cap(\partial \Lambda \times I)$ is a circle. To get a con- 
tradiction, suppose that $J$ is such a component. Let $F_{J}$ be the component of $F$ such that $J \subset \partial F_{J}$. We claim that $J$ is contractible in $\partial \Lambda \times I$. Since $h(\partial \Lambda \times I) \subset N-\operatorname{Fr}(N)$, it follows that $h(J) \subset D$. Hence $h(J)$ is contractible in $W-K$. Since $P-K$ is incompressible in $W-K$, it follows that $h(J)$ is not homotopic in $W-K$ to $P \cap \operatorname{Fr}(N)$. Therefore $J$ is not parallel in $\partial \Lambda \times I$ to $\partial \Lambda \times 0$. It follows that $J$ is contractible in $\partial \Lambda \times I$.

Since $J$ is contractible in $\Lambda \times I$ and since $\pi_{1}\left(F_{J}\right) \rightarrow \pi_{1}(\Lambda \times I)$ is injective, it follows that $F_{J}$ is a disk. There is a 3-ball $B_{J}$ in $\Lambda \times I$ such that $\partial B_{J}=F_{J} \cup\left(B_{J} \cap(\partial \Lambda \times I)\right)$. We may choose $J$ so that $B_{J} \cap F=F_{J}$. By using case (2) of Lemma 6.5 of [He], we may reduce $\sharp(F)$. This is a contradiction.

Lemma 6. Let $g: \Lambda \times I \rightarrow W-K$ be a proper map that agrees with $f$ on $\Lambda \times \partial I$. Let $F=g^{-1}(\operatorname{Fr}(M))$. Suppose that $F$ is properly embedded and 2 -sided in $\Lambda \times I$. Suppose that if $F^{\prime}$ is a component of $F$, then $F^{\prime}$ is not a 2-sphere and $\pi_{1}\left(F^{\prime}\right) \rightarrow \pi_{1}(\Lambda \times I)$ is injective.

(1) If $F^{\prime}$ is a component of $F$, then $F^{\prime}$ is either a disk or an annulus. Furthermore, at least one component $A$ of $F$ is an annulus that meets $\Lambda \times 0$ in a single circle that is noncontractible in $\Lambda \times 0$.

(2) If no component of $F \cap(\partial \Lambda \times I)$ is a circle, then either $g(\partial \Lambda \times I) \subset$ $M-\operatorname{Fr}(M)$ or $\sharp(\partial \operatorname{cl}(Q-f(\Delta \times 1)) \cap D)$ can be reduced by an isotopy of $W$ that is fixed on $K \cup \operatorname{cl}(W-N)$.

(3) If $g(\partial \Lambda \times I) \subset M-\operatorname{Fr}(M)$, then $\partial F \subset \Lambda \times \partial I$ and either $F=A$ or $\sharp(Q \cap \operatorname{Fr}(M))$ can be reduced by an isotopy of compact support fixed on $K \cup g(\partial \Lambda \times I)$.

Proof. To prove (1), let $F^{\prime}$ be a component of $F$. Since $\pi_{1}(\Lambda \times I)=\mathbb{Z}$, it follows that $F^{\prime}$ is either a disk, annulus, or Möbius band. Note that $\Lambda \times I$ contains no 2-sided Möbius band. Let $A$ be the component of $F$ that contains the unique component of $F \cap(\Lambda \times 0)$. Since $P-K$ is incompressible, it follows that $A$ is not a disk.

To prove (2), suppose that no component of $F \cap(\partial \Lambda \times I)$ is a circle and that $g(\partial \Lambda \times I)$ is not contained in $M-\operatorname{Fr}(M)$. Since $g(\partial \Lambda \times I)$ is not contained in $M-\operatorname{Fr}(M)$, it follows that $F \cap(\partial \Lambda \times I)$ is nonempty. Let $\alpha$ be a component of $F \cap(\partial \Lambda \times I)$. Then $\alpha$ is an arc. Since $g(\partial \Lambda \times 0) \subset M-\operatorname{Fr}(M)$, it follows that $\partial \alpha \subset \partial \Lambda \times 1$. Let $D_{\alpha} \subset \partial \Lambda \times I$ be the disk that is separated off by $\alpha$. Let $\beta=D_{\alpha} \cap(\partial \Lambda \times 1)$ and let $\beta^{\prime}=g(\beta)$. We may choose $\alpha$ so that $D_{\alpha} \cap F=\alpha$.

Now $g(\alpha) \subset D$. Let $h=g \mid D_{\alpha}$. Then exactly one of the following manifolds contains $h\left(D_{\alpha}\right)$ :

(1) $M-K$ or

(2) $\operatorname{cl}(N-M)$. 
Let $\Omega$ be whichever of these two manifolds contains $h\left(D_{\alpha}\right)$. Let $\Theta$ be the result of splitting $\Omega$ along $Q \cap \Omega$.

Recall that $D_{\alpha} \subset \partial \Lambda \times I$. We lose nothing by assuming that $g$ is such that, for some $\varepsilon>0, g(\partial \Lambda \times[1-\varepsilon, 1])$ lies all on one side of a regular neighborhood of $Q$ in $W$. Consequently, $h^{-1}(Q)$ is the union of $\beta$ and circles that are in the interior of $D_{\alpha}$. We may modify $h$ so that $h^{-1}(Q)=\beta$. Consequently, we may assume that $h\left(D_{\alpha}\right)$ is contained in $\Theta$. Note that $h \mid \alpha$ is fixed endpoint homotopic in $D$ to an arc. We may therefore assume that $h\left(\partial D_{\alpha}\right)$ is a circle in $\partial \Theta$. Let $B$ be a regular neighborhood of $h\left(D_{\alpha}\right)$ in $\Theta$. Then, by the Loop Theorem, there is a disk $D^{\prime} \subset B$ such that $\partial D^{\prime}$ is nontrivial in $B \cap \partial \Theta$. Since $B \cap \partial \Theta$ is an annulus, we may we may assume that $\partial D^{\prime}=h\left(\partial D_{\alpha}\right)$. Consequently, $D^{\prime} \cap D=\alpha$ and $D^{\prime} \cap Q=\beta^{\prime}$. Let $B^{\prime}$ be a regular neighborhood of $D^{\prime}$ in $W$ such that $B^{\prime} \cap \Omega=B$.

We may use $B^{\prime}$ to reduce $\sharp(\partial \operatorname{cl}(Q-f(\Delta \times 1)) \cap D)$ by an isotopy of $W$ fixed on $K \cup \operatorname{cl}(W-N)$.

To prove (3), suppose that $g(\partial \Lambda \times I) \subset M-\operatorname{Fr}(M)$. Then $F \cap(\partial \Lambda \times I)=\emptyset$ and so $F-(\Lambda \times 0) \subset \Lambda \times 1$. Now suppose that $F \neq A$. Let $G$ be a component of $F-A$. Then $\partial G \subset \Lambda \times 1$.

Suppose that $G$ is a disk. Then $g(\partial G)$ is contractible in $\operatorname{Fr}(M)$. Therefore there is a disk $G^{\prime} \subset \operatorname{Fr}(M)$ with $\partial G^{\prime}=g(\partial G)$. We may assume that $G^{\prime} \cap Q=$ $\partial G^{\prime}$. Let $G^{\prime \prime}$ be the unique disk in $Q$ with $\partial G^{\prime \prime}=\partial G^{\prime}$. Since $Q-K$ is incompressible in $W-k$, it follows that $G^{\prime \prime} \subset W-K$. So $G^{\prime} \cup G^{\prime \prime}$ is a 2-sphere in $W-K$ that bounds a 3-ball $U^{\prime}$ in $W-K$.

On the other hand, suppose that $G$ is an annulus. By standard arguments, there is a parallelism $U \subset \Lambda \times I$ with $\partial U=G \cup(U \cap(\Lambda \times 1))$. Then, by Proposition 5.4 of [Wa], there is a parallelism $U^{\prime}$ in $W-K$ between $g(U \cap(\Lambda \times 1))$ and a 2-manifold in $\operatorname{Fr}(M)$.

In either case, we may use $U^{\prime}$ to reduce $\sharp(Q \cap \operatorname{Fr}(M))$ by an isotopy of compact support fixed on $K \cup g(\partial \Lambda \times 1)$.

\section{The main theorem}

TheOREM 7. There is an isotopy $q_{t}: W \rightarrow W$ such that $q_{1}(Q)=P$.

Proof. By Lemma 1 of [W], there is a compact, connected 3-manifold $V_{1}$ that traps both $P$ and $Q$ and is such that $P \cap V_{1}$ is a single disk and no component of $\operatorname{Fr}\left(V_{1}\right)$ is a 2-sphere. Let $\Delta \subset \mathbb{R}^{2}$ be a disk and let $\Lambda=\operatorname{cl}\left(\mathbb{R}^{2}-\Delta\right)$. Choose $\Delta$ so that $f(\Lambda \times I)$ is contained in $W-V_{1}$. By Lemma 3 there is a compact, connected 3-manifold $V_{1}^{\prime} \subset W$ with $V_{1} \subset V_{1}^{\prime}-\operatorname{Fr}\left(V_{1}^{\prime}\right)$ such that $f(\Delta \times I) \subset V_{1}^{\prime}-\operatorname{Fr}\left(V_{1}^{\prime}\right), V_{1}^{\prime} \cap P$ is a disk, and $V_{1}^{\prime}$ can be compressed in $W-V_{1}$ to a compact 3-manifold $X_{1}^{\prime}$ by removing 1-handles that miss $P$. Let $X_{1}$ be the component of $X_{1}^{\prime}$ that contains $V_{1}$. 
Then $V_{1} \subset X_{1}-\operatorname{Fr}\left(X_{1}\right)$ and $\operatorname{Fr}\left(X_{1}\right)$ is incompressible in $W-V_{1}$. Let $D=\operatorname{cl}\left(\operatorname{Fr}\left(X_{1}\right)-\operatorname{Fr}\left(V_{1}^{\prime}\right)\right)$.

Let $h$ be an isotopy of $W$ that has compact support. Let $\widehat{f}: \mathbb{R}^{2} \times I \rightarrow W$ be defined by $\widehat{f}(x, t)=h_{t}(f(x, t))$. Then $\widehat{f}\left(\mathbb{R}^{2} \times 0\right)=P$; put $\widehat{f}\left(\mathbb{R}^{2} \times 1\right)=Q_{1}$. Let $a=\sharp\left(\partial \operatorname{cl}\left(Q_{1}-\widehat{f}(\Delta \times 1)\right) \cap D\right)$ and let $b=\sharp\left(Q_{1} \cap \operatorname{Fr}\left(X_{1}\right)\right)$. Suppose that $h$ is fixed on $V_{1}$ and that $h_{t}(f(\Delta \times I)) \subset V_{1}^{\prime}-\operatorname{Fr}\left(V_{1}^{\prime}\right)$ for every $t \in I$. Choose $h$ among such isotopies so that $(a, b)$ is minimal in lexicographic order.

Let $g: \Lambda \times I \rightarrow W-V_{1}$ be a proper map that agrees with $\widehat{f}$ on $\Lambda \times \partial I$. Let $F=g^{-1}\left(\operatorname{Fr}\left(X_{1}\right)\right)$. By Lemma 5 we may choose $g$ so that if $F^{\prime}$ is a component of $F$, then $F^{\prime}$ is not a 2-sphere and the inclusion induced map $\pi_{1}\left(F^{\prime}\right) \rightarrow \pi_{1}(\Lambda \times I)$ is injective, and so that no component of $F \cap(\partial \Lambda \times I)$ is a circle.

By Lemma 6(2) and the minimality of $a$, it follows that $g(\partial \Lambda \times I) \subset$ $X_{1}-\operatorname{Fr}\left(X_{1}\right)$. By Lemma $6(3)$ and the minimality of $b$, it follows that $F$ is a single annulus with $F \cap(\Lambda \times i)$ a single circle that is noncontractible in $\Lambda \times i$ for $i \in \partial I$. Therefore $g \mid F$ is a homotopy in $\operatorname{Fr}\left(X_{1}\right)$ between $P \cap \operatorname{Fr}\left(X_{1}\right)$ and $Q_{1} \cap \operatorname{Fr}\left(X_{1}\right)$, which are both single circles. Therefore $P \cap \operatorname{Fr}\left(X_{1}\right)$ is isotopic in $\operatorname{Fr}\left(X_{1}\right)$ to $Q_{1} \cap \operatorname{Fr}\left(X_{1}\right)$. Without loss of generality, we may assume that $P \cap \operatorname{Fr}\left(X_{1}\right)$ is parallel to $Q_{1} \cap \operatorname{Fr}\left(X_{1}\right)$ in $\operatorname{Fr}\left(X_{1}\right)$ by applying an isotopy of $W$ that is fixed off a product neighborhood of $\operatorname{Fr}\left(X_{1}\right)$.

Let $V_{2}$ be a compact, connected 3-manifold that contains $V_{1}^{\prime}$ and the support of $h$. We may choose $V_{2}$ so that $P \cap V_{2}$ is a single disk, so that $\operatorname{Fr}\left(V_{2}\right)$ contains no 2-spheres, and so that $V_{2}$ contains any prechosen compact subset of $W$. As before, we may construct a plane $Q_{2}$ that is isotopic to $Q_{1}$ by an isotopy of compact support fixed on $V_{2}$ and a compact, connected 3-manifold $X_{2}$ such that $Q_{2} \cap \operatorname{Fr}\left(X_{2}\right)$ and $P \cap \operatorname{Fr}\left(X_{2}\right)$ are single circles that are parallel in $\operatorname{Fr}\left(X_{2}\right)$.

Continuing in this fashion, we may construct an exhaustion $X$ and a plane $Q^{\prime}$ isotopic to $Q$ so that, for $n \in \mathbb{N}, P \cap \operatorname{Fr}\left(X_{n}\right)$ and $Q^{\prime} \cap \operatorname{Fr}\left(X_{n}\right)$ are single circles that are parallel to one another in $\operatorname{Fr}\left(X_{n}\right)$. Let $h_{t}^{\prime}: W \rightarrow W$ be the isotopy that takes $Q$ to $Q^{\prime}$. Define $f^{\prime}: \mathbb{R}^{2} \times I \rightarrow W$ by $f^{\prime}(x, t)=h_{t}^{\prime}(f(x, t))$. To conserve notation, put $f=f^{\prime}$ and $Q=Q^{\prime}$.

For $n \in \mathbb{N}$, let $M_{n}=\operatorname{cl}\left(X_{n+1}-X_{n}\right)$, let $A_{n}=P \cap M_{n}$ and $B_{n}=Q \cap M_{n}$. By standard arguments, there is an isotopy of $M_{n}$ fixed on $\partial M_{n}$ that takes $B_{n}$ to an annulus $B_{n}^{\prime}$ such that each component of $A_{n} \cap B_{n}^{\prime}$ is a circle in $M_{n}-\operatorname{Fr}\left(M_{n}\right)$ that is noncontractible in $M_{n}$. We may compose these isotopies for each $n \in \mathbb{N}$ so that we may assume that each component of $A_{n} \cap B_{n}$ is a circle that is noncontractible in $M_{n}$.

We may construct another exhaustion $Y$ for $W$ as follows. Let $Y_{1}=X_{1}$. By Lemma 4, there is a compact, connected 3-manifold $Y_{2}$ that contains $X_{2}$, 
meets $P$ and $Q$ in single disks whose boundaries are parallel in $\operatorname{Fr}\left(Y_{2}\right)$, and can be compressed in $W-Y_{1}$ to a compact 3-manifold $Z_{1}^{\prime}$ by removing 1-handles that miss both $P$ and $Q$. Let $Z_{1}$ be the component of $Z_{1}^{\prime}$ that contains $Y_{1}$. Since $P \cap \operatorname{Fr}\left(Y_{2}\right)$ is noncontractible in $W-Y_{1}$, it follows that none of the compressing 1-handles removed from $Y_{2}$ to obtain $Z_{1}^{\prime}$ intersected the parallelism in $\operatorname{Fr}\left(Y_{2}\right)$ between $P \cap \operatorname{Fr}\left(Y_{2}\right)$ and $Q \cap \operatorname{Fr}\left(Y_{2}\right)$.

Continuing in the obvious way, we may construct an exhaustion $Y$ for $W$ such that for $n \in \mathbb{N}$,

(1) $Y_{n}$ is connected,

(2) $P \cap Y_{n}$ and $Q \cap Y_{n}$ are disks whose boundaries are parallel in $\operatorname{Fr}\left(Y_{n}\right)$,

(3) $Y_{n+1}$ can be compressed in $W-Y_{1}$ by removing 1-handles that miss $P$ and $Q$ to obtain $Z_{n}^{\prime}$, and

(4) if $Z_{n}$ is the component of $Z_{n}^{\prime}$ that contains $Y_{1}$, we may assume that $Z_{n} \subset Z_{n+1}-\operatorname{Fr}\left(Z_{n+1}\right)$. (This is because $\operatorname{Fr}\left(Z_{n}\right)$ is incompressible in $W-Y_{1}$.)

Note that properties (1)-(4) are preserved under the taking of subsequences.

Let $\Delta \subset \mathbb{R}^{2}$ be a disk and let $\Lambda=\operatorname{cl}\left(\mathbb{R}^{2}-\Delta\right)$. Choose $\Delta$ so that $f(\Lambda \times I) \subset W-Y_{1}$. By taking a subsequence of $Y$ and the corresponding subsequence of $Z$, we may assume that $f(\Delta \times I) \subset Y_{2}-\operatorname{Fr}\left(Y_{2}\right)$.

Let $n \geq 1$ be an integer. By Lemma 5, there is a map $g: \Lambda \times I \rightarrow W-Y_{1}$ that agrees with $f \mid \Lambda \times I$ on $\partial(\Lambda \times I)$ such that if $F_{n}=g^{-1}\left(\operatorname{Fr}\left(Z_{n}\right)\right)$ and if $F^{\prime}$ is a component of $F_{n}$, then $F^{\prime}$ is not a 2 -sphere and $\pi_{1}\left(F^{\prime}\right) \rightarrow \pi_{1}(\Lambda \times I)$ is injective. By parts (1) and (2) of Lemma 6 , it follows that $F_{n}$ is a single annulus.

It is not difficult to see that we may warp the product structure of $\Lambda \times I$ so that $F_{n}=J \times I$ for some circle $J \subset \Lambda$. Let $\Lambda^{\prime}$ be the closure of the component of $\Lambda-J$ that has noncompact closure. Arguing as before, there is a map $g^{\prime}: \Lambda^{\prime} \times I \rightarrow \operatorname{cl}\left(W-Z_{n}\right)$ that agrees with $g \mid \Lambda^{\prime} \times I$ on $\partial\left(\Lambda^{\prime} \times I\right)$ such that $\left(g^{\prime}\right)^{-1}\left(\operatorname{Fr}\left(Z_{n+1}\right)\right)$ is a single annulus $F_{n+1}$. We may warp the product structure of $\Lambda^{\prime} \times I$ off $\partial \Lambda^{\prime} \times I$ so that $F_{n+1}=J^{\prime} \times I$ for some circle $J^{\prime} \subset \Lambda^{\prime}$ that is parallel in $\Lambda$ to $J$. Let $A \subset \Lambda$ be the annulus with $\partial A=J \cup J^{\prime}$. Put $N_{n}=\operatorname{cl}\left(Z_{n+1}-Z_{n}\right)$ and $M_{n}=\operatorname{cl}\left(Z_{n+1}-Y_{n+1}\right)$. Then $g^{\prime} \mid A \times I: A \times I \rightarrow N_{n}$ is a homotopy from $P \cap N_{n}$ to $Q \cap N_{n}$.

By an isotopy of $W$ fixed off a product neighborhood of $\bigcup_{n \in \mathbb{N}} \operatorname{Fr}\left(Y_{n}\right)$, we may assume that $P \cap \operatorname{Fr}\left(Y_{n}\right)=Q \cap \operatorname{Fr}\left(Y_{n}\right)$. By composing this isotopy with the appropriate homotopies, we retain that $P \cap \operatorname{Fr}\left(Y_{n}\right)$ is homotopic to $Q \cap N_{n}$ in $N_{n}$ for every $n \in \mathbb{N}$. By Lemma 2 , it follows that $P \cap N_{n}$ is isotopic in $M_{n}$ by an isotopy fixed on $\partial M_{n}$. By piecing together these isotopies, we may assume that $P \cap \operatorname{cl}\left(W-Z_{1}\right)=Q \cap \operatorname{cl}\left(W-Z_{1}\right)$ and that $P \cap Z_{1}$ and $Q \cap Z_{1}$ are disks that share a common boundary. Since $W$ is irreducible, $(P \cap Q) \cap Z_{1}$ bounds a ball $B$. Use $B$ to finish isotoping $Q$ onto $P$. 


\section{References}

[BT] M. G. Brin and T. L. Thickstun, 3-manifolds which are end 1-movable, Mem. Amer. Math. Soc. 411 (1989).

[BBF] E. M. Brown, M. S. Brown and C. D. Feustel, On properly embedding planes in 3-manifolds, Trans. Amer. Math. Soc. 55 (1976), 461-464.

[BF] E. M. Brown and C. D. Feustel, On properly embedding planes in arbitrary 3-manifolds, Proc. Amer. Math. Soc. 94 (1985), 173-178.

[He] J. Hempel, 3-manifolds, Ann. of Math. Stud. 86, Princeton Univ. Press, 1976.

[Wa] F. Waldhausen, On irreducible 3-manifolds which are sufficiently large, Ann. of Math. 87 (1968), 56-88.

[W] B. N. Winters, Properly homotopic, nontrivial planes are parallel, Topology Appl. 48 (1992), 235-243.

DEPARTMENT OF MATHEMATICS

PITTSBURG STATE UNIVERSITY

PITTSBURG, KANSAS 66762

U.S.A.

E-mail: WINTERS@MAIL.PITTSTATE.EDU

Received 25 May 1993;

in revised form 27 June 1994 